\title{
Influence of the surface pressure on the organization of mixed Langmuir films of behenic acid and glucose oxidase
}

\author{
J.-M. Chovelon, ${ }^{1}$ M. Provence, ${ }^{2}$ N. Jaffrezic-Renault, ${ }^{2}$ V. Derue, ${ }^{3}$ D. Lair, ${ }^{3}$ S. Alexandre ${ }^{3}$ \\ and J.-M. Valleton ${ }^{3, *}$ \\ ${ }^{1}$ Laboratoire d'Application de la Chimie à l'Environnement (LACE), UMR 5634 CNRS / Univ. Lyon 1, Université \\ Claude Bernard Lyon 1, 69622 Villeurbanne Cedex (France) \\ ${ }^{2}$ Laboratoire d'Ingénierie et de Fonctionnalisation des Surfaces (IFoS), UMR 5621 CNRS / ECL, Ecole Centrale de \\ Lyon, BP 163, 69131 Ecully Cedex (France) \\ ${ }^{3}$ Laboratoire "Polymères, Biopolymères, Membranes" (PBM), UMR 6522 CNRS / Univ. Rouen, Université de Rouen, \\ 76821 Mont-Saint-Aignan Cedex (France)
}

\begin{abstract}
The mechanism of adsorption of glucose oxidase at the air-water interface and under a behenic acid film has been studied. Fluorescence microscopy shows that glucose oxidase molecules adsorb at the air-water interface to form circular domains. At the equilibrium state, coalescence of the glucose oxidase domains occurs leading to a homogeneous Gibbs film of enzyme. When behenic acid is present at the air-water interface, the increase in surface pressure due to the glucose oxidase adsorption is faster than with a behenic acid free air-water interface. Isothermal compression expansion cycles of the mixed glucose oxidase behenic acid film were performed. This study shows that glucose oxidase molecules are adsorbed under the behenic acid film and at the air-water interface. Insertion of glucose oxidase molecules at the air-water interface occurs when the surface pressure of the film is below $25 \mathrm{mN} / \mathrm{m}$. When the surface pressure is above $35 \mathrm{mN} / \mathrm{m}$, the inserted glucose oxidase molecules are expelled from the air-water interface.
\end{abstract}

Keywords: behenic acid, glucose oxidase, Langmuir films, surface pressure

\section{INTRODUCTION}

Langmuir-Blodgett (LB) films have been proposed for various applications because of their thickness of molecular dimensions [1,2]. A great challenge is the functionalization of $\mathrm{LB}$ films by the incorporation of proteins. Since a protein is able to recognize a specific molecule, this would allow the formation of a mixed LB film with specific molecular recognition properties. Such specificity is of great interest in the field of biosensors [3-6].

Biosensors are based on coupling a biomolecule able to specifically recognize a chemical or biochemical species to a transducer, which converts the physicochemical signal coming from the host-molecule interactions into an electrical signal. Biosensors based on LB technology use an active film comprising one or several layers of the amphiphilic molecule into which the enzyme is incorporated. In comparison with biosensors made using polymeric films, the extremely low thickness of LB films will lead to a decrease of the response time of the biosensor. Moreover, the LB technique allows a better control of the amount of proteins in the active film.

*Author for correspondence.
With the aim of building a biosensor based on LB technology, the behaviour at the air-water interface of a mixed film of glucose oxidase (GOx) and behenic acid has been studied [7]. After transfer, the resulting mixed LB films were characterized by IR spectroscopy $[8,9]$ and atomic force microscopy [9-12]. These studies allowed us to describe the formation of the mixed film. GOx molecules are incorporated in the behenic acid at low pressure and are gradually adsorbed under the behenic acid monolayer.

In this paper, we present new results on the influence of the surface pressure on the incorporation of GOx molecules in the mixed film. Compression/ expansion cycles of the mixed film were performed under various conditions in order to better understand the incorporation mechanism of GOx in the film. The adsorption of glucose oxidase at the air-water interface was visualized by fluorescence microscopy.

\section{MATERIALS AND METHODS}

GOx from Aspergillus niger is a homodimeric protein, characterized by a molecular weight of 155000 , and an isoelectric point of 4.2 , it was obtained from Sigma (Type X-S) and used without further 
purification. Behenic acid (99\%) was provided by Sigma. The fluorescence probe, Texas Red, was purchased from Molecular Probes (Engene, Ovegm). The water used was purified using a Millipore system (Milli-RO and Milli-Q units) involving reverse osmosis, deionization, an active charcoal cartridge, and filtration. Its $\mathrm{pH}$ was about 5.6.

Langmuir experiments were carried out with a Langmuir trough from Nima (type 611). The dimensions of the trough were $30 \mathrm{~cm} \times 20 \mathrm{~cm}$, the volume was $500 \mathrm{~cm}^{3}$. This system used two mobile barriers for compressing the molecules and a Wilhelmy balance for measuring the interfacial pressure. The trough and the barrier are made of PTFE. In order to limit evaporation, the trough was placed in a cabinet.

The subphase temperature was held at $22 \pm 1{ }^{\circ} \mathrm{C}$ during the experiments. The subphase was constituted of water or GOx aqueous solutions at different concentrations. Before each experiment, the surface of the subphase (water or GOx aqueous solutions) was cleaned by aspiration. The surface was considered as cleaned when the change in surface pressure of the clean subphase upon compression of the area was less than $0.2 \mathrm{mN} / \mathrm{m}$. The end of surface cleaning was chosen as the origin of time for all experiments. Behenic acid was spread from chloroform solution onto the subphase using a $100 \mu \mathrm{l}$ micropipette. The spreading was achieved at the same distance above the subphase in all experiments.

For studiyng evolution of the surface pressure, its change was recorded until a constant value was obtained.

Pressure-area isotherms were made by compressing the film at a constant barrier speed of $0.5 \mathrm{~cm} / \mathrm{min}$. When the subphase was a GOx solution, the recording of the pressure-area isotherm was started after a delay of $45 \mathrm{~min}$.

Compression/expansion cycles were performed for pure behenic acid and for mixed films. The cycles were carried out at a constant barrier speed of $1 \mathrm{~cm} / \mathrm{min}$. The monolayers were compressed to a fixed surface pressure value and then decompressed up to the maximum area of the trough before recompression. The recompression was started either immediately after the decompression (pure behenic acid or mixed films) or after a fixed surface pressure was reached (mixed film). Another series of compression/expansion experiments was carried out in which decompression was stopped at a fixed surface pressure before an immediate recompression.

A small laboratory-build trough was used to follow the protein adsorption by fluorescence microscopy. The microscope was an inverted microscope (Axiovert 135, Zeiss, Germany) equipped with a water immersion objective. The objective was placed in the subphase and focused on the interface. The GOx molecules were fluorescently labelled with Texas Red according to the procedure described by J.A.Titus et al. [13]. A filter facilitated the excitation and observation of Texas Red-labelled GOx molecules at the interface.

\section{RESULTS AND DISCUSSION}

\subsection{Adsorption of GOx at the air-water interface}

Before studying the formation of the mixed film of behenic acid and GOx, it was important to have information on the effect of the enzyme alone on the air-water interface. It is well known that enzymes are amphiphilic molecules and therefore may form a film at the air-water interface. The adsorption of enzyme molecules at the air-water interface was followed by measuring the surface pressure and by fluorescence microscopy.

The surface pressure of a GOx solution increases with time. This observation shows that molecules of GOx are adsorbed at the air-water interface. We can see, from figure 1 , that the increase in surface

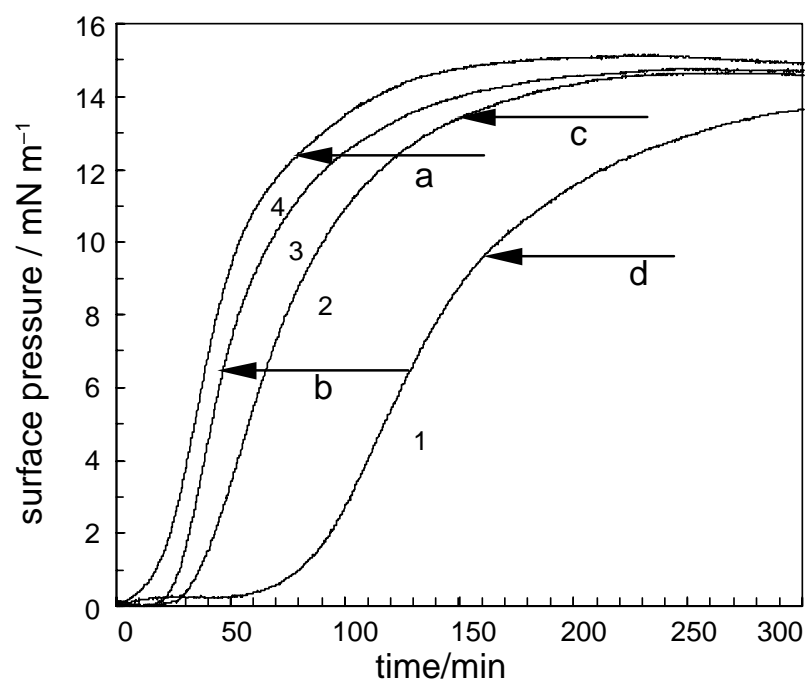

Figure 1.Time evolution of the surface pressure for different concentrations of GOx. (1) : $1.6 \mathrm{mg} / \mathrm{l} ;$ (2) : $3.2 \mathrm{mg} / \mathrm{l} ;(3): 4.8$ $\mathrm{mg} / \mathrm{l} ;(4): 6.2 \mathrm{mg} / \mathrm{l}$. The lettered arrows on curve 2 correspond to the fluorescence microscopy images taken during the adsorption of GOx molecules at the air-water interface (see figure 2).

pressure is time and concentration dependent. The surface pressure starts to rise after $5 \mathrm{~min}$ for the highest enzyme concentration $(6.2 \mathrm{mg} / \mathrm{l})$, after $30 \mathrm{~min}$ for the intermediate enzyme concentration $(3.2 \mathrm{mg} / \mathrm{l})$ and after $60 \mathrm{~min}$ for the lowest enzyme concentration $(1.6 \mathrm{mg} / \mathrm{l})$, implying that, formation of the GOx film at the air-water interface is faster at the highest concentration than at the lowest 
concentrations. After this increase, the surface pressure tends to become constant, the system reaches an equilibrium state. The time to obtain the equilibrium pressure is dependent on the initial GOx concentration $(250 \mathrm{~min}$ for the higher enzyme concentrations and $360 \mathrm{~min}$ for the lowest concentration). Increasing the GOx concentration in the subphase reduces the time necessary to reach the equilibrium pressure. This pressure is about $15 \mathrm{mN} / \mathrm{m}$ for all the concentrations used. At this value, it may be assumed that the protein adsorption at the air-water interface is maximal. Studies on the adsorption of GOx at the air-water interface have already been reported in the literature. Rosilio et al. also found that the kinetics of adsorption of GOx were dependent on GOx concentration in the subphase [14]. However the adsorption profiles they obtained were different from those presented here. As suggested from additional experiments, these differences may be due to differences in the geometry of the trough used for the experiments or to thermal convection due to temperature gradients.

In order to understand how the adsorption of GOx occurs at the air-water interface, we followed the adsorption of GOx by fluorescence microscopy (figure 2). For this study, the GOx molecules were

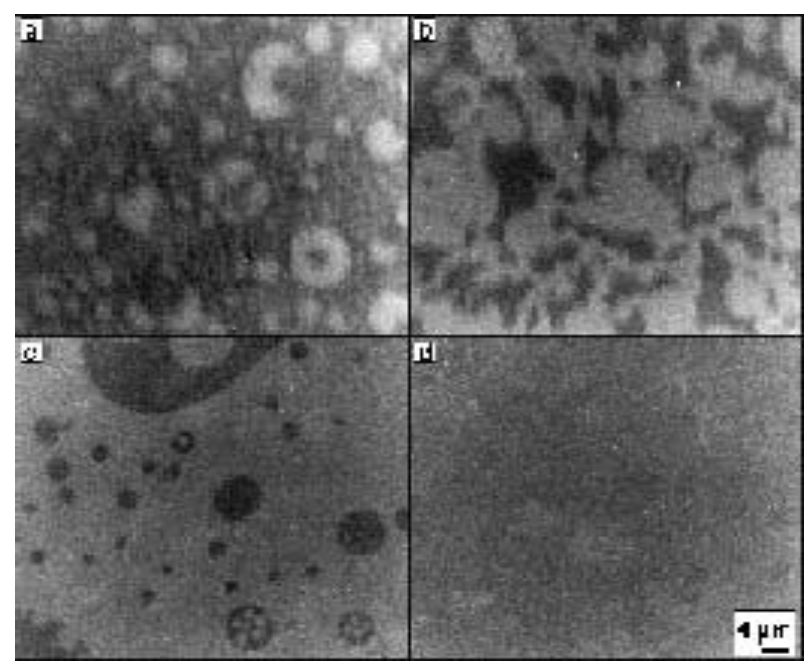

Figure 2. Adsorption of GOx at the air-water interface observed by fluorescence microscopy. The GOx molecules were labelled with Texas Red. The 4 images a, b, c, d were taken at different stages of adsorption corresponding to surface pressures indicated by arrows on figure 1 .

labelled with a fluorescent probe. In the first minutes, we observed quasi-circular domains of labelled GOx (image a). The size of these domains increased with time and the domains began to coalesce (image b). On the image c (advanced stage of the adsorption process), a few dark circular domains are still visible. Inside these domains, small circular domains of labelled GOx can be observed. The last dark domains disappear progressively and a continuous Gibbs film of GOx is then observed (image d). However a few regions with a more intense fluorescence can be observed, which may be due to the formation of GOx aggregates, or to reorganization of the 3D structure of GOx.

\subsection{Mixed films of behenic acid and GOx}

Influence of behenic acid on the adsorption of GOx

In order to study the influence of behenic acid on the adsorption of GOx, surface pressure versus time curves were performed for different behenic acid concentrations (figure 3 ). The curve for pure GOx is

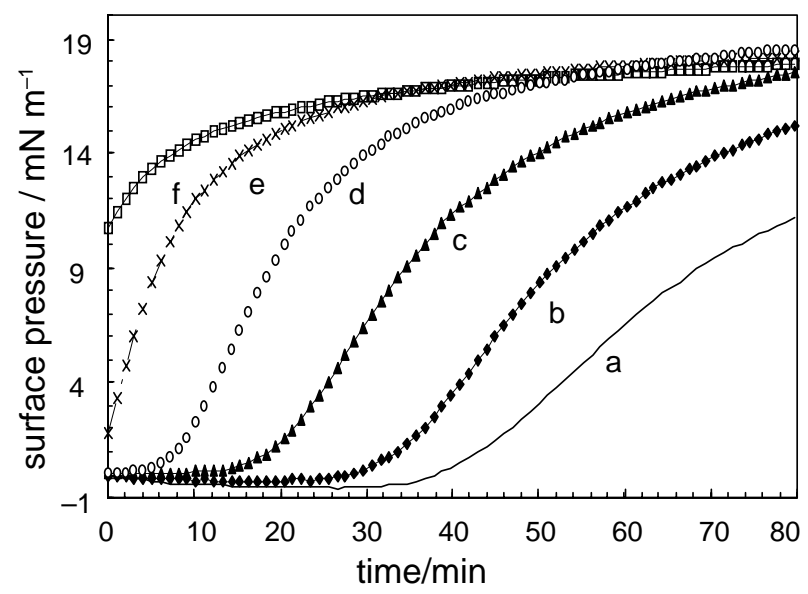

Figure 3. Effect of the behenic acid concentration at the air-water interface on the adsorption of GOx (3.2 mg/l). (a): absence of behenic acid molecules at the air water interface. (b-f): behenic acid concentrations corresponding to molecular areas of: (b) : $130.3 \AA^{2}$; (c) : $65.2 \AA^{2}$; (d) : $43.4 \AA^{2}$; (e) : $32.6 \AA^{2}$; (f) : $29 \AA^{2}$

shown as a reference (curve a). Clearly, we can see that the time to reach the adsorption equilibrium of GOx depends on the behenic acid concentration. For behenic acid concentrations corresponding to molecular areas between 29 and $43.4 \AA^{2} /$ molecule, it is necessary to wait 40 to $50 \mathrm{~min}$ to reach the adsorption equilibrium of GOx while for lower concentrations, 60 to $200 \mathrm{~min}$ are necessary. Equilibrium is reached for a surface pressure of $17 \mathrm{mN} / \mathrm{m}$ whatever the concentration of behenic acid. When behenic acid was not present at the interface, equilibrium was reached after $250 \mathrm{~min}$. The adsorption of GOx resulted in a faster increase in surface pressure with behenic acid than without it, the rapidity increasing with the amount of behenic acid concentrations.

\section{Pressure-area isotherms}

In the previous study [7], it was deduced a way to elaborate mixed films at the air-water interface for a GOx solution with a concentration of $3.2 \mathrm{mg} / \mathrm{l}$ was determined. After spreading the behenic acid solution at a concentration corresponding to a 
molecular area of $43.4 \AA^{2} /$ molecule, an adsorption time of 45 min was necessary to reach the equilibrium surface pressure of $16 \mathrm{mN} / \mathrm{m}$ and then to start the isotherm recording (figure $4 \mathrm{a}$ ). The pressure-area

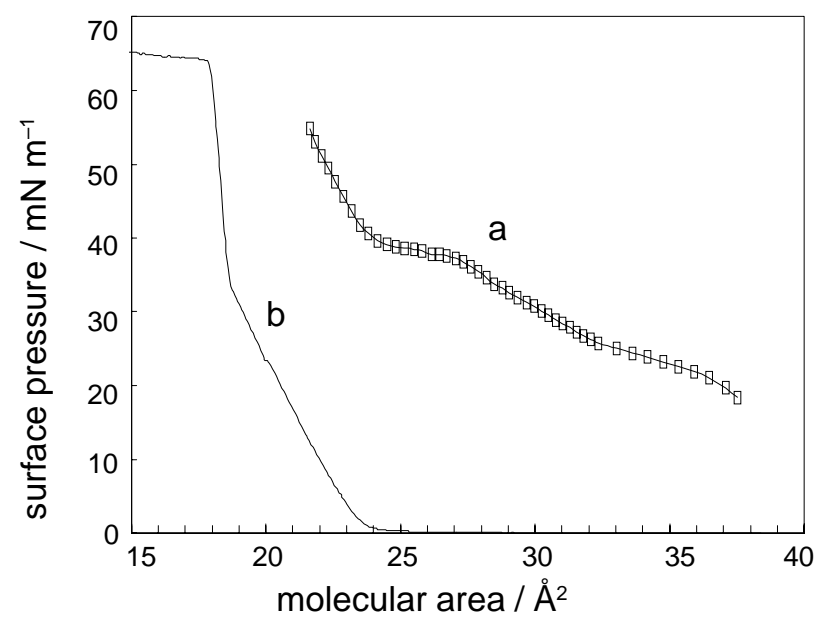

Figure 4. Pressure-area isotherms of a pure monolayer of behenic acid (a) and a mixed film obtained with a concentration of GOx of $3.2 \mathrm{mg} / \mathrm{l}$ in the subphase and a surface concentration of behenic acid corresponding to a molecular area of $43 \AA^{2}$ (b). The recording was done after a relaxation time of $45 \mathrm{~min}$, sufficient to allow adsorption equilibrium of GOx at the air-water interface, to be attained.

isotherm of behenic acid on pure water was shown as a reference (figure 4b). For the mixed film, an apparent molecular area was defined as the ratio of the film area to the number of behenic acid molecules. We observe that when GOx is present in the subphase, the apparent molecular area is greater than for the pure behenic acid layer at the same surface pressure. This reveals that molecules of GOx are inserted between behenic acid molecules at the air-water interface. A transition is observed on the isotherm at around $38 \mathrm{mN} / \mathrm{m}$. This transition may indicate rearrangement towards higher molecular ordering, expulsion of enzyme molecules from the interface into the bulk, or coverage of enzyme molecules by the behenic acid film [15]. As soon as the surface pressure reaches $40 \mathrm{mN} / \mathrm{m}$, the slope of the isotherm increases significantly but stays always lower than that of pure behenic acid. This may indicate either the continuous expulsion of GOx molecules from the interface or a higher compressibility of the mixed film compared to a pure behenic acid film. The phase transitions at $22 \mathrm{mN} / \mathrm{m}$ and $32 \mathrm{mN} / \mathrm{m} \mathrm{[16]} \mathrm{observed}$ when the subphase is pure water are difficult to observe when GOx is present in the subphase. Dubreuil et al. [7] obtained pressure-area isotherms of the mixed films of GOx and behenic acid with various concentrations of GOx in the subphase. They observed these phase transitions for the lower concentrations of GOx. The isotherm obtained by these authors, with a GOx concentration of $3.2 \mathrm{mg} / \mathrm{l}$, shows a lower apparent molecular area whatever the surface pressure, compared to the isotherm presented here. In that work, recording of the isotherm started after the equilibrium surface pressure was reached, thus the number of enzyme molecules at the air-water interface or under the behenic acid film is greater, which can explain the higher apparent molecular area observed.

\section{Compression /decompression experiments}

In order to be able to enterpret the shape of the pressure-area isotherms, compression/expansion cycles were performed for the mixed film. The monolayer was compressed to a surface pressure of $55 \mathrm{mN} / \mathrm{m}$ and then decompressed up to the maximum area of the trough.

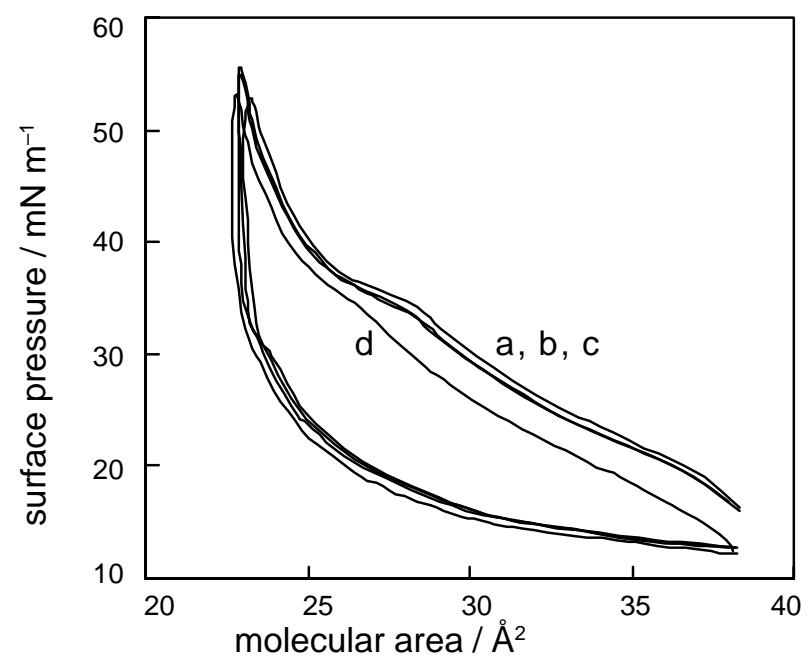

Figure 5. Isotherms of mixed behenic acid/GOx films under compression and expansion. (a): first cycle, surface pressure of $16 \mathrm{mN} / \mathrm{m}$ before compression; (b): second cycle, recompression after again reading the initial surface pressure of $16 \mathrm{mN} / \mathrm{m}$;

(c): third cycle, recompression after again reading the initial surface pressure of $16 \mathrm{mN} / \mathrm{m}$; (d): fourth cycle, immediate recompression.

Four cycles (figure 5) were carried out at a constant barrier speed of $1 \mathrm{~cm} / \mathrm{min}$. A delay of $45 \mathrm{~min}$ before starting was required in order to reach the adsorption equilibrium of GOx. The surface pressure before compression was $16 \mathrm{mN} / \mathrm{m}$ and after the first cycle, at the end of the expansion cycle, it was $12 \mathrm{mN} / \mathrm{m}$. We then waited until the initial surface pressure of $16 \mathrm{mN} / \mathrm{m}$ was reached again before starting the second cycle. The third cycle also started after the initial surface pressure of $16 \mathrm{mN} / \mathrm{m}$ was reached. We can see that the shapes of isotherms (a), (b), (c) are similar. For the fourth cycle, an immediate recompression was performed. So the surface pressure before compression was $12 \mathrm{mN} / \mathrm{m}$ instead of $16 \mathrm{mN} / \mathrm{m}$. In this case, we can observe that the 
pressure-area isotherm is shifted to lower areas. This series of compression/expansion experiments shows that the shape of the mixed film isotherm depends on the initial surface pressure value and thus of the quantity of GOx present at the air-water interface. The difference in surface pressure before the compression and at the end of the expansion cycle may be explained by the expulsion, during compression, of GOx molecules from the interface into the bulk. When the recompression is immediate, the GOx molecules do not have enough time to be adsorbed again. Nevertheless some GOx molecules are adsorbed during decompression. Consequently the number of GOx molecules at the air-water interface is smaller and the apparent molecular area is lower whatever the surface pressure. Lie et al. [17] observed a similar behaviour when they studied the compression/expansion properties of monolayers of dimyris toylphosphatidylcholine(DMPC), dipalmitoylphosphatidylcholine (DPPC) and distearoylphosphatidylcholine (DSPC) mixed with $\beta$-casein at the air-water interface. Their results showed that the protein was squeezed out upon compression but re-entered the mixed monolayer upon expansion. For the mixed monolayer of behenic acid and GOx, Dubreuil et al. demonstrated that $28 \mathrm{mN} / \mathrm{m}$ was the limit pressure of incorporation of the enzyme in the film at the air-water interface [7].

Other series of compression/expansion experiments were carried out in order to limit the readsorption of GOx molecules during the expansion cycle (figure 6). In this case, the film was compressed to a surface pressure of $45 \mathrm{mN} / \mathrm{m}$ and then decompressed down to surface pressures of 15 (curve b), 20 (curve c) and $25 \mathrm{mN} / \mathrm{m}$ (curve d) before recompression. Isotherm (a) corresponds to the mixed film isotherm obtained after a GOx adsorption time of $45 \mathrm{~min}$. In this isotherm the transition at $38 \mathrm{mN} / \mathrm{m}$ is observed, not visible on the other isotherms. Isotherm (b), obtained after expansion of the mixed film down to $15 \mathrm{mN} / \mathrm{m}$, shows a phase transition at $32 \mathrm{mN} / \mathrm{m}$. This pressure corresponds to that of the phase transition of a pure behenic acid film between the liquid condensed and the solid phases. In isotherm (c), obtained after expansion of the mixed film down to $20 \mathrm{mN} / \mathrm{m}$, it can be seen that the slope between 23 and $31 \mathrm{mN} / \mathrm{m}$ is significantly higher than the slope of the initial isotherm (a). Furthermore a phase transition at $22 \mathrm{mN} / \mathrm{m}$ is observed, the pressure corresponding to that of the phase transition of a pure behenic acid film between the two liquid condensed phases. In isotherm (d), obtained after expansion of the mixed film up to $25 \mathrm{mN} / \mathrm{m}$, we note that the compressibility in the condensed regions between 25 and $32 \mathrm{mN} / \mathrm{m}$, and between 32 and 45 $\mathrm{mN} / \mathrm{m}$, is close to that of behenic acid. As stated before, the phase transition at $38 \mathrm{mN} / \mathrm{m}$ may indicate the expulsion of GOx molecules from the interface. The lack of this transition on the isotherms obtained during the second compression suggests that all the
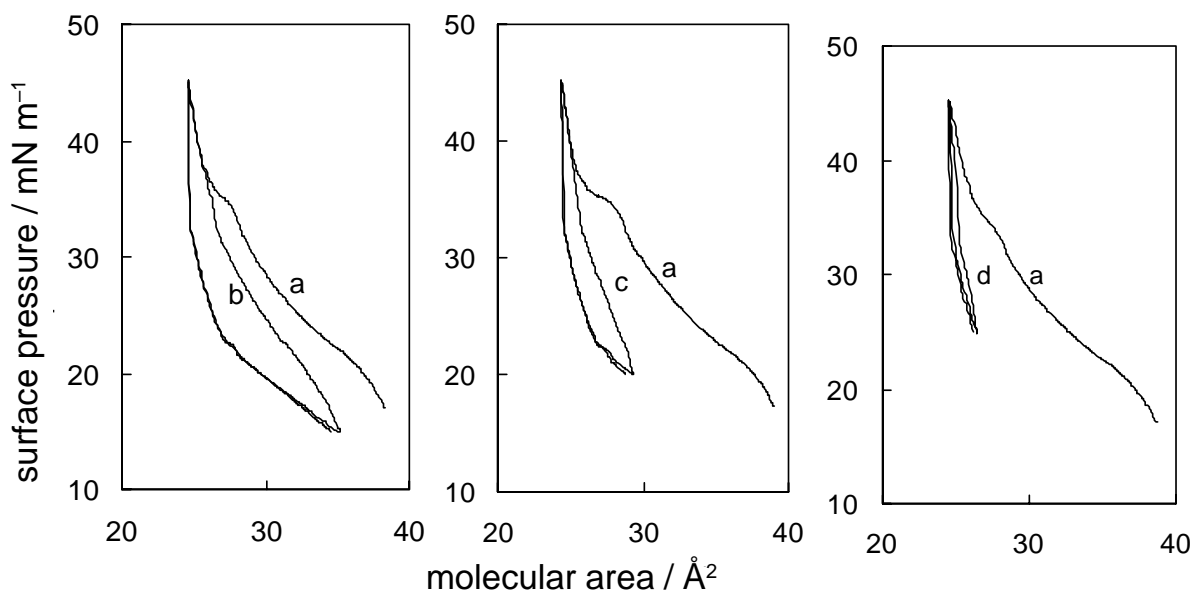

Figure 6. Isotherms of mixed behenic acid/GOx films under compression and expansion. (a) initial isotherm; (b-d): compression isotherms obtained after expansion of the film down to surface pressure of: (b) $15 \mathrm{mN} / \mathrm{m}$; (c) $20 \mathrm{mN} / \mathrm{m}$; (d) $25 \mathrm{mN} / \mathrm{m}$.

GOx molecules were expelled from the interface at the first compression and no GOx molecules were adsorbed during decompression. As decompression was stopped at a fixed surface pressure, the GOx molecules do not have enough time to be adsorbed again. Moreover the film is in a condensed state probably preventing the incorporation of $\mathrm{GOx}$ molecules at the air-water interface. Consequently only behenic acid molecules would remain at the airwater interface before the second compression. However, at the second compression the area per behenic acid molecule is greater than the area that could be expected from the pressure-area isotherm of pure behenic acid. This implies that the GOx 
molecules are present beneath the behenic acid film. The distance between the behenic acid molecules may be increased because of behenic acid/enzyme interactions [18].

Furthermore the compression/expansion isotherms of the mixed film are not reversible and hysteresis is observed. For example, in figure 6, for a surface pressure of $30 \mathrm{mN} / \mathrm{m}$, the apparent molecular area is equal to $30 \AA^{2}$ during the compression cycle, whereas a value of $25 \AA^{2}$ was found during the expansion cycle. Since all the GOx molecules are expelled from the interface, we could expect that, as observed for the monolayers of pure behenic acid, the isothermal compression/expansion would be reversible. This lack of reversibility is a complementary proof of the presence of GOx molecules under the behenic acid film. This phenomenon could be due to time dependent reorganization of the film. When enzymes are present under the behenic acid film, interactions take place and during expansion of the barrier,a longer time is required to dissociate the complexes formed, thus explaining the strong hysteresis behaviour.

Model of the evolution of the mixed film

The evolution of the mixed film at the air-water interface when the surface pressure is increased may be described as follows (figure 7):

For surface pressures between 2 and $16 \mathrm{mN} / \mathrm{m}$, GOx molecules are adsorbed at the interface (stage (2)). Whatever the behenic acid concentration, an equilibrium is reached at around $16 \mathrm{mN} / \mathrm{m}$ (figure 3). We assumed that at this value, protein adsorption at the air-water interface was maximal, and all the isotherms were started after this surface pressure was reached. A concentration of behenic acid corresponding to a molecular area of $43 \AA^{2} /$ molecule and a concentration of GOx of 3.2 $\mathrm{mg} / \mathrm{l}$ were chosen. It has been noted in the literature that for low concentrations of GOx $(1.2 \mathrm{mg} / \mathrm{l})$ the mixed monolayers of behenic acid and GOx exhibit the same phase transitions as for the pure behenic acid monolayer [7]. This suggests that the incorporation of GOx into the behenic acid monolayer does not change the monolayer phases of the behenic acid and that behenic acid and GOx are localized at the air-water interface into different domains. Hence the mixed monolayer would be constituted of behenic acid domains and GOx domains. For a concentration of behenic acid corresponding to a molecular area of $43.4 \AA^{2}$, the behenic acid film is at a phase transition between the gaseous and the liquid condensed phases. Then the GOx molecules can adsorb at the air-water interface in the domains of gas phase. In the domains of liquid condensed phase, the available space is too

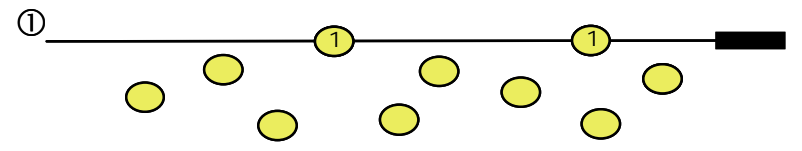

(2)

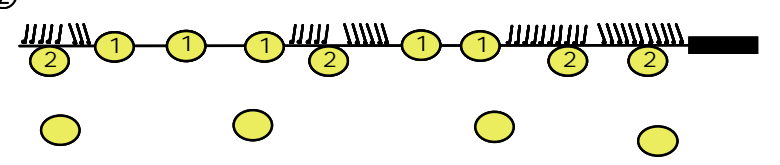

(3)
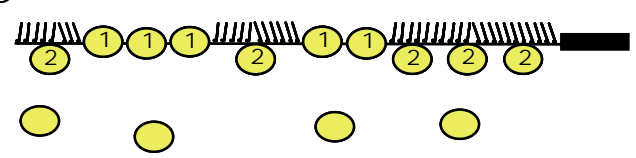

(4)

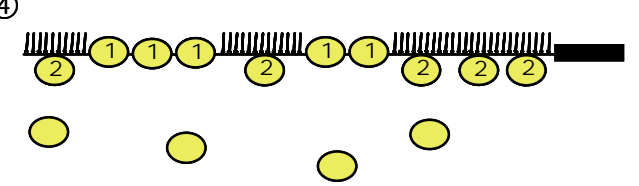

(5)

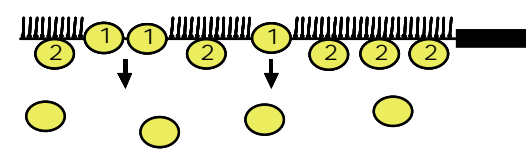

(6)

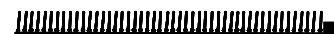
(2) (2) (2) (2) (2)

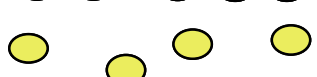

1 Behenic acid molecules

GOx molecules in the subphase

(1) Domains of GOx molecules at the interface

(2) GOx molecules adsorbed under the behenic acid film

Figure 7. Model of the evolution of the mixed behenic acid/GOx film at the air-water interface. Stage (1): before the spreading of the behenic acid solution, the surface pressure is about $2 \mathrm{mN} / \mathrm{m}$ showing that some GOx molecules are already adsorbed at the air-water interface. Stage (2): After $45 \mathrm{~min}$, the surface pressure has reached a maximum of $16 \mathrm{mN} / \mathrm{m}$ due to the adsorption of GOx molecules at the air-water interface (molecules labeled 1) and under the behenic acid film (molecules labeled 2). Stage (3): Compression of the mixed GOx / behenic acid film from a surface pressure of 16 to $32 \mathrm{mN} / \mathrm{m}$; the behenic acid molecules are in a liquid condensed phase. Stage (4): Further compression of the mixed film up to $38 \mathrm{mN} / \mathrm{m}$; the behenic acid molecules are then in a solid phase. Stage (5): At a surface pressure of about $38 \mathrm{mN} / \mathrm{m}$ the GOx molecules are expelled from the air-water interface. Stage (6): For the high surface pressure (above $38 \mathrm{mN} / \mathrm{m}$ ) the mixed film is constituted of a behenic acid monolayer in a solid phase under which interact GOx molecules. 
small to allow enzyme penetration. So the GOx molecules cannot adsorb at the air-water interface in the condensed domains but only under the condensed domains of behenic acid. Indeed the compression/ expansion experiments showed that the GOx molecules were well present under the behenic acid film. Consequently, it is likely that at this surface pressure $(16 \mathrm{mN} / \mathrm{m})$ proteins are present at the air-water interface, and some of them are already in contact with behenic acid.

As soon as the surface pressure of $16 \mathrm{mN} / \mathrm{m}$, is reached, the compression can start (stage (3) to (4)). The increase of the surface pressure leads to a closer approach of enzymes and behenic acids. In the second series of compression/expansion experiments, we limited the readsorption of GOx molecules during the expansion cycle. Then we clearly observed during the phase transitions characteristic of behenic acid at the second compression: in particular, the phase transition at $22 \mathrm{mN} / \mathrm{m}$ which corresponds to the transition between two liquid condensed phases and the transition at $32 \mathrm{mN} / \mathrm{m}$ characteristic of the liquid condensed-solid phase transition. So it is likely that for the surface pressures from 16 to $32 \mathrm{mN} / \mathrm{m}$, the behenic acid film is in a liquid condensed state while for values pressures higher than $32 \mathrm{mN} / \mathrm{m}$, the behenic acid film is in a solid state.

For surface pressures between 37 and $40 \mathrm{mN} / \mathrm{m}$ (stage (5), we can see a transition which is not visible on the isotherm of pure behenic acid (figure 4). This transition may indicate some reorganization of the mixed film. We may assume that for such surface pressure values, enzyme molecules are expelled from the interface and pushed under the behenic acid film. As a consequence, the surface pressure does not increase significantly while the molecular area decreases.

Above $40 \mathrm{mN} / \mathrm{m}$ (stage (6), the slope of the isotherm increases significantly but remains lower than those for pure behenic acid. In this case, it is possible to consider a higher compressibility of the mixed film compared to pure behenic acid. Moreover, the apparent molecular area of behenic acid is higher than that obtained with the pure behenic acid film (with no GOx molecules in the subphase). This is an additional proof that GOx molecules interact with behenic acid.

\section{SUMMARY \& CONCLUSIONS}

The adsorption of labeled GOx molecules at the air-water interface has been observed by fluorescence microscopy. In a first time, circular domains of labelled GOx are formed at the air-water interface.
Finally, these circular domains coalesce and a homogeneous film of labeled GOx is obtained.

When behenic acid molecules are spread at the air-water interface, we observed a faster increase in surface pressure due to the GOx adsorption than when the GOx molecules adsorb directly at the airwater interface. Pressure area isotherms show an increase in the apparent molecular area of the behenic acid molecules when GOx molecules are present in the subphase. This indicates that GOx molecules are inserted at the air-water interface.

Isothermal compression/expansion cycles of the mixed GOx / behenic acid film were performed. They show that during the compression, the GOx molecules inserted at the air-water interface in the behenic acid film are expelled from the interface at a surface pressure of $35 \mathrm{mN} / \mathrm{m}$. After expansion of the film to surface pressure values lower than $20 \mathrm{mN} / \mathrm{m}$, glucose oxidase molecules readsorb back at the airwater interface. This is not the case when the film is expanded to a surface pressure of $25 \mathrm{mN} / \mathrm{m}$, in that case no hysteresis is observed, showing that GOx molecules no longer adsorb at the air-water interface, presumably due to the compactness of the film. However, in all cases, GOx molecules also interact with the polar head of the behenic acid molecules leading to a shift of the pressure area isotherm to the higher molecular areas for all surface pressure values.

These results allowed us to present a model of formation of the mixed film at the air-water interface. In a first time GOx molecules adsorb at the air-water interface and interact with the behenic acid film. When the film is compressed the GOx molecules inserted in the film are expelled from the air-water interface at a surface pressure of about $38 \mathrm{mN} / \mathrm{m}$. Then the mixed film is constituted of GOx molecules interacting with the behenic acid monolayer, interactions which lead to an increase in the molecular area of behenic acid.

The conditions for obtaining high quality Langmuir-Blodgett films containing glucose oxidase can be chosen from this analysis of the structure and the stability of the corresponding mixed film formed at the air-water interface.

\section{REFERENCES}

1. Roberts, G. Langmuir-Blodgett Films, Plenum: New York, (1990).

2. Petty, M.C. Thin Solid Films 210/211 (1992) 417.

3. Arisawa, S., Arise, T., and Yamamoto, R. Thin Solid Films 209 (1992) 259.

4. Fiol, C., Valleton, J.-M., Delpire, N., Barbey, G., Barraud, A., and Ruaudel-Teixier, A. Thin Solid 
Films, 210/211 (1992) 489.

5. Zaitsev, S.U., Colloids Surf. A : Physicochem. Eng. Asp. 75 (1993) 211.

6. Collings, A.F. and Caruso, F. Rep. Prog. Phys.60 (1997) 1397.

7. Dubreuil, N., Alexandre, S., Fiol, C. and Valleton, J.-M. Journal of Colloid and Interface Science 181 (1996) 393.

8. Fiol, C., Alexandre, S., Dubreuil, N. and Valleton, J.M. Thin Solid Films 261 (1995) 287.

9. Alexandre, S., Dubreuil, N., Fiol, C., Lair, D., Sommer, F., Tran Minh Duc, and Valleton, J.-M. Thin Solid Films 293 (1992) 295.

10.Dubreuil, N., Alexandre, S., Fiol, C., Sommer, F. and Valleton, J.-M. Langmuir 11 (1995) 2098.

11.Sommer, F., Alexandre, S., Dubreuil, N., Lair, D., Tran-Min Duc and Valleton, J.-M. Langmuir 13 (1997) 791.

12. Dubreuil, N., Alexandre, S., Lair, D., Sommer,
F., Tran-Min Duc and Valleton, J.-M. Colloids Surf. B: 11 (1998) 95.

13.Titus, J.A., Haugland, R., Sharrow, S. O. and Segal, D. M. J.Immunological Methods 50 (1982) 193.

14.Rosilio, V., Boissonnade, M. M., Zhang, J., Jiang, L. and Baszkin, A. Langmuir 13 (1997) 4669.

15. Schawarz, G. Ber.Bunsenges. Phys. Chem. 100 (1996) 999.

16.Kehn, R. M., Böhm, C., Bibo, A. M., Peterson, I. R., Möhwald, H., Nielsen, J. A. and Kjaer, K. J. Phys. Chem. 95 (1991) 2092.

17. Lie, J. B., Krägel, J., Makievski, A. V., Fainermann, V. B., Miller, R. and Möhwald, H. Colloids Surf. A: 142 (1998) 355.

18. Vikholm, I., and Teleman, O. J. Colloid Interface Sci. 168 (1994) 125. 